\title{
Uncalm before the storm
}

Cite as: CMAJ 2020 April 14;192:E417-8. doi: 10.1503/cmaj.1095860

Posted on cmajnews.com on March 27, 2020

$\mathbf{T}$ hese are anxious days for most people, but especially for the health workers who are preparing for worse still to come as cases of coronavirus disease 2019 (COVID-19) multiply across Canada. Some health care workers are balancing long hours at work with coordinating childcare and supports for elderly parents. Other have chosen to isolate themselves to keep loved ones safe if they get sick, given dwindling supplies of protective equipment. Still others worry about the heart-wrenching decisions they will likely have to make when there are more patients than ventilators.

Dr. Mamta Gautam, a psychiatrist who specializes in physician wellness, has heard colleagues refer to what they're experiencing now as "pre-TSD." Recently, she started holding daily video chats to offer peer support.

"We have such vivid and harrowing information coming out from other countries. There's the sense that this is the calm before the storm [in Canada] and we're steeling ourselves for the next couple of weeks," she says.

\section{Preparing for crisis}

Worries can seem impossible to turn off, so Gautam recommends giving them 20 minutes a day. "Ruminating is not productive when it's going on past that," she says.

Now is the time to tend to any "holes in our energy tank" by figuring out what tasks can be delegated or dropped, Gautam adds. "Maybe that playroom doesn't have to be cleaned up," she suggests. Health workers need to make sure they are filling their tanks, too, by setting up routines and paying attention to relationships that give them energy, whether that's taking a walk or chatting with a friend.

According to Dr. Robert Maunder, head of research in Mount Sinai Hospital's department of psychiatry, the anticipation of traumatic events is "almost always worse than the actual exposure" because people tend to focus on worst-case scenarios. During the 2003 severe acute respiratory syndrome (SARS) outbreak, $18 \%$ to $57 \%$ of health workers experienced substantial emotional distress, but very few experienced lasting psychological harm.

In a recent video about how to cope with COVID-19, he and fellow psychiatrist Dr. Jon Hunter encouraged colleagues to focus on and remind themselves of the things they can control.

"There is so much of this situation that's not in the individual's control," Maunder explains. But there are also many things within every health professional's power. For example, he suggests, "I have been well trained. I know the hand-washing protocol. I know how to don and doff my personal protective equipment."

At Mount Sinai, Maunder and his colleagues are offering mental health rounds, expanding drop-in hours for health workers and training peer supporters in every department.

\section{Weathering the storm}

Kindness and compassion are paramount in a crisis. According to Maunder, many of the stresses that emerged during the 2003 SARS pandemic were "interpersonal," with communication breaking down between frontline workers, managers and leaders. "Everybody is working tired, so they get grumpy with each other," he says.

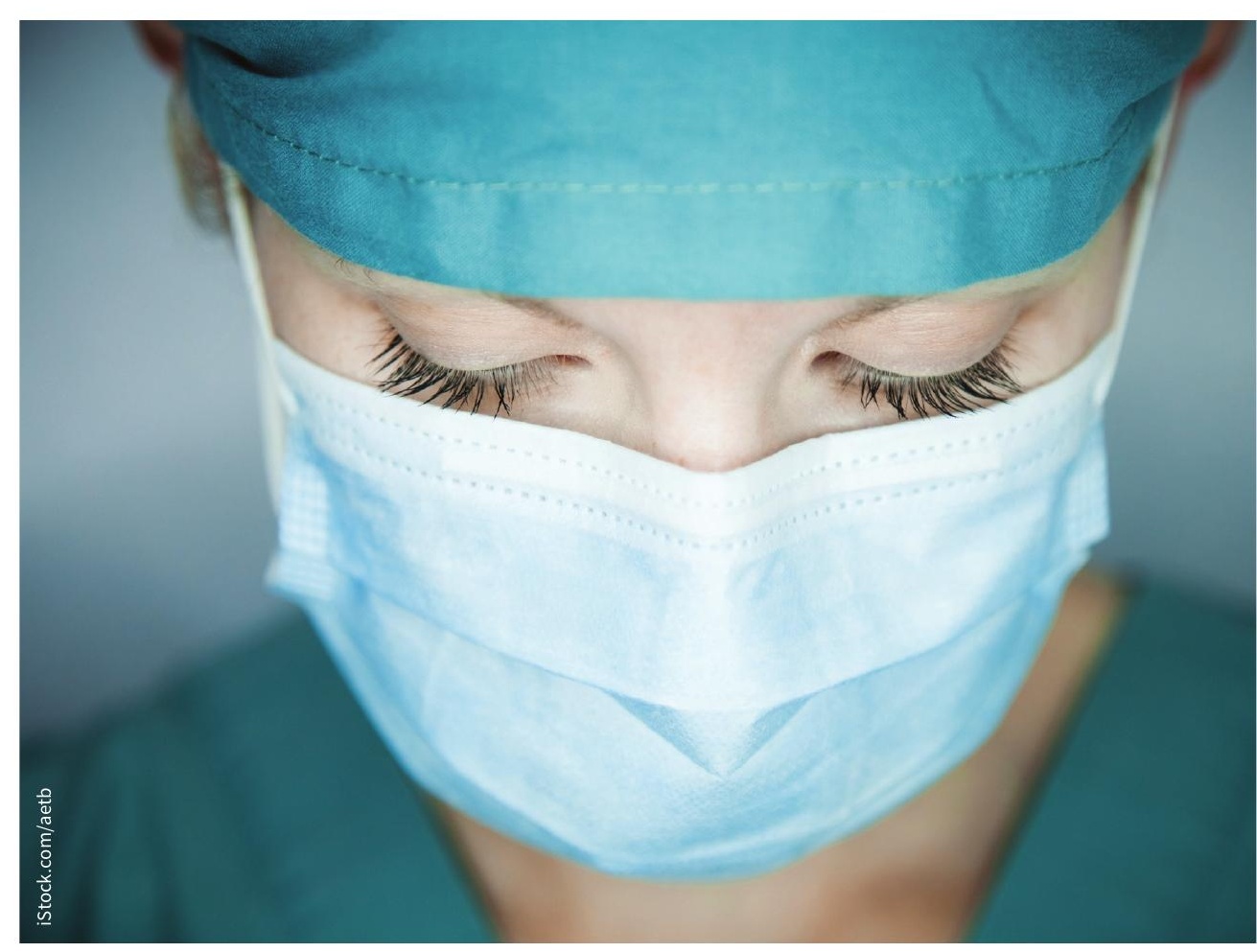

Canada's COVID-19 outbreak is not yet at its peak, but its psychological impact is already being felt among health workers. 
According to Gautam, it's important to remember that decision makers and colleagues are doing their best as the situation changes from one hour to the next. "We're all human beings caring for other human beings," she says, and focusing on that truth builds connection and soothes resentment.

Health workers may also find it helpful to remember why they chose their profession, and to remind their colleagues, too. "The heart and the passion are what help people come in every day. That's what we need to tap into now as we move forward together," Gautam says.

Maunder calls this "finding meaning." Tapping into the bigger picture of solidarity with colleagues and compassion for patients are key to weathering extremely difficult events, he says.

It's normal when a situation becomes overwhelming to ask questions like "What's the point?" Maunder says the answers to those questions "always bring us back to our values."

\section{In the aftermath}

Dr. Sandro Galea, dean of the Boston University School of Public Health, has written extensively on the populationwide mental health effects of what he calls "unexpected, large, traumatic events," such as Hurricane Katrina and SARS. Affected populations experience higher rates of depression, substance use and posttraumatic stress disorder for months and even years after the fact, he said.

Some health workers won't notice psychological effects until the pandemic is over. "In the short term, there are adjustments to the stressor," Galea explains. As health workers focus on meeting the immediate physical needs of themselves, their families and their patients they often consciously push aside their emotional responses. But, once the crisis passes, suppressed fear, sadness, guilt and anger can come to the fore.

This delay in response is why patients aren't diagnosed with PTSD until at least one month after exposure to trauma,
Galea explains. "We have to wait for the adjustment to pass."

For this reason, services to support health workers should continue after a crisis has passed, and advocates must build awareness that long-term effects are expected, he says.

Normalizing the psychological effects of a crisis can make it easier for health workers to seek help. In a study Maunder and colleagues conducted of health workers in Toronto who responded to the SARS outbreak, those who felt adequately trained and supported in their workplace were less likely to be diagnosed with a psychiatric disorder in the years after.

Long-term, system-wide solutions - like avoiding over-scheduling and efforts to reduce violence in the emergency department - are also vital to help health workers weather stressful periods, Maunder says. "The most impactful solutions will not be individual ones, but systemic ones."

Wendy Glauser, Toronto, Ont. 